ASE-17-0063.R1

Research Report

\title{
What do the public know about anatomy? Anatomy education to the public and the implications
}

\author{
Adam M. Taylor ${ }^{1 *}$, Peter Diggle ${ }^{2}$, Quenton Wessels ${ }^{3}$ \\ ${ }^{1}$ Clinical Anatomy Learning Centre, Lancaster Medical School, Lancaster University, Lancaster, \\ United Kingdom \\ ${ }^{2}$ Centre for Health Informatics, Computing and Statistics, Lancaster Medical School, Lancaster \\ University, Lancaster, United Kingdom \\ ${ }^{3}$ Department of Anatomy, School of Medicine, University of Namibia, Windhoek, Namibia
}

Running Title: Anatomical knowledge of the general public

Correspondence to: Dr. Adam M. Taylor, Lancaster Medical School, Faculty of Health and Medicine, Lancaster University, Bailrigg, Lancaster, LA1 4YB. United Kingdom.

E-mail: a.m.taylor@lancaster.ac.uk 


\begin{abstract}
Public knowledge of the anatomical "self" is lacking and evidence points towards a growing need for anatomy education to the wider public. The public were offered the opportunity to learn human anatomy and complete an anatomical knowledge survey afterwards. Sixty-three participants volunteered to attempt to place 20 anatomical structures on a blank human body template. Responses were scored independently and then collated. A mixed effects logistic model was used to examine any associations with participants' as a random effect and all other factors as fixed effects. Results showed a statistically significant quadratic trend with age. Participants in health-related employment scored significantly higher than those not in healthrelated employment. There was a significant interaction between gender and organ type with males scoring higher than females in identifying muscles, but not in identifying internal organs. The current study demonstrates the general public's eagerness to learn anatomy despite their limited knowledge of the human body, and the need for widening participation. Furthermore, it raises an awareness of the anatomical literacy needs of the general public, especially in school children and young adults. Furthermore, it emphasizes the value of health literacy as a focus in undergraduate medical education. Anatomy literacy appears to be neglected, and this experience provides an example of a possible mode of public engagement in anatomy.
\end{abstract}

Keywords: gross anatomy education, medical education, public education, public engagement, anatomy outreach programs, widening participation 


\section{INTRODUCTION}

The general public's fascination with the structure and function of the human body coincides with the history of medicine, particularly anatomical dissections. Cadaveric dissections gradually gained popularity as a result of the scientific revival in medieval Italy at the beginning of fourteenth century (Gosh, 2015). Anatomical instruction, particularly the work of Italian physicians and anatomists Mondino de Luzzi (ca. 1270-1326) at Bologna, and Andreas Vesalius (1514-1564) at Padua, evolved to become public events for all to learn (Ferrari, 1987; Gosh, 2015). The persistent large crowds drawn by anatomy museums of the Victorian medical landscape and the modern day "Body Worlds" exhibits bears witness to society's interest in anatomy (Goeller, 2007; Bates, 2008). "Body Worlds" is a public exhibition demonstrating various parts of the human anatomy from deceased individuals (human and other species) that have been plastinated to preserve them. "Body Worlds" raises many ethical and moral questions, but its stated intended use is to engage the public and enable them to learn about the body (Morriss-Kay, 2002; Fonseca and Finn, 2016). Public engagement in anatomical teaching and exhibits provide an opportunity to assess the existing knowledge gap with the aim to facilitate health literacy, demonstrate the importance of sound anatomical knowledge to doctors and healthcare professionals and improve the targeting and efficiency of health campaigns. Previous studies have assessed the knowledge of the general public before and after visiting anatomical exhibitions such as Body Worlds, demonstrating a statistically significant increase in anatomical knowledge after visiting such an exhibit (Jones, 2002; Goeller, 2007; Fonseca and Finn, 2016). There is an opportunity to mirror public engagement and interest in anatomical learning with education, which can serve as a tool for improving the general population's experience when focusing on their health and communicating with medical professionals. 
General knowledge of the anatomical "self" is lacking and has far-reaching implications for health literacy as outlined in the following section. Health literacy has been defined as the ability to "obtain, process, and understand health information and services needed to make appropriate health decisions" (Nielsen-Bohlman et al., 2004). Health literacy has become one of the core competencies in undergraduate medical education (Williams et al., 1998; Kripalani and Weiss, 2006; GMC, 2009, 2015). Anatomical literacy is taken for granted due to the part and parcel nature of the use of anatomical terms in medicine. Body knowledge in patients visiting certain specialty clinics and in the general population has been assessed, demonstrating that even individuals who have acute medical problems associated with individual organs cannot reliably demonstrate the location of the affected organ above the level of knowledge of the general population. The exceptions to this trend relates to individuals with liver or kidney problems (Weinman et al., 2009, Bernhard et al., 2016). Patients' lack of knowledge is not surprising given that it is not their profession to know this material; however it does aid in diagnosis when making initial contact with a healthcare professional. It has been shown that medical terminology is not well understood by the general public (Lerner et al., 2000). Further complications in understanding have been shown to arise from medical professionals over-estimating the knowledge-level of their patients (Kelly and Haidet, 2007). The impact of inadequacies in health literacy is far-reaching and forms a major barrier that hinders effective treatment. For instance, patients with chronic diseases such as diabetes and hypertension require patient education in order to monitor their conditions and achieve adequate control (Williams et al., 1998). In subjects that are "anatomy heavy" such as medicine, the need for anatomical knowledge is essential (Drake et al., 2009). The sheer breadth and depth of anatomical knowledge required of students is vast and assessment driven learning is inevitable, particularly when the weighting of 
anatomy within the exam is considered (Wormald, 2009). It is important however to highlight that assessment of anatomy is linked to the purpose, i.e. to future competent clinical practice (Smith and McManus, 2015). The breadth and depth of anatomical knowledge for competent future practice will depend upon the chosen specialty, but should always be greater than that of the patients they serve.

Plain language communication is a major challenge for many healthcare providers due to their use of medical terminology that has become part and parcel of their profession. A recent poll by Prostate Cancer UK found more than 50\% of the 2,000 men they surveyed did not know the anatomical location of the prostate, $17 \%$ of the individuals were unaware they had a prostate and only $8 \%$ knew its function (Fiore, 2016). This leads one to question the efficacy of contemporary health campaigns across the globe where the target audience lacks basic anatomical knowledge. Public health campaigns raise awareness of the public around pertinent health issues such as smoking and lung cancer in the hope to improve health and reduce the need for medical treatment for morbidities associated with certain lifestyle choices. There are questions that should be asked such as would these campaigns be necessary, or perhaps more effective if more was taught about the human body in outreach and educational programs at schools? Evidence from the US has already demonstrated that there is a demand for knowledge on the human body from young students but teachers are often not equipped with the resources or knowledge to delivery relevant material (Baram-Tsabari and Yarden, 2007; Burns, 2008; Burns, 2012). Growing evidence points towards the demand for anatomy courses for members of the public (Gillingwater and Findlater, 2015). This in turn has the potential to form a facet of patient education initiatives, specifically health education which aims to improve the health behaviors of individuals, groups or communities (Whitehead, 2004). Lancaster Medical School, as part of 
Lancaster University's "Campus in the City" initiative and public engagement, recently provided the public with the opportunity to view the human body in three-dimensions (3D). This provided the authors with an opportunity to showcase the fascinating field of anatomy, assess the public's knowledge and perceptions of anatomy and anatomical structures, and demystify erroneous concepts. The aim of this study was to provide an interactive platform for the general public to learn anatomy. Furthermore, the study also assessed their knowledge on the location of various organs of the human body and examined trends associated with age, gender, education, work in healthcare settings and recent visit to a healthcare professional.

\section{MATERIALS AND METHODS}

During the spring of 2016 members of the public in the City of Lancaster had the opportunity to attend a "Campus in the City (CITC)". This was setup in an empty retail outlet which was branded with University logo and advertising. The CITC represented an opportunity to engage the public in the various academic endeavors of Lancaster University. Over ten weeks the academic content of the CITC changed. The event utilized for this public engagement and research was the "human body" event, organized by two anatomists and held over four days attracting four hundred and fifty nine visitors. Attendees were given the opportunity to learn more about human anatomy and if interested the opportunity to complete an anatomical knowledge survey. An opportunity was provided to explore any structure of interest, which was then projected in 3D via a Cyber-Anatomy Med ${ }^{\mathrm{TM}}$ software (Cyber-Anatomy Corp., Coralville, IA) connected to an Optoma GT 1080 short throw projector (Optoma Europe Ltd, Watford, UK). The software package provided the participants with an opportunity to discover more than 4,300 high resolution digital structures and over 13,000 identifiable landmarks. Participants also had 
the opportunity to explore the structures of the human body through anatomical models, including; a detailed torso and heart, a skull, an articulated skeleton, and a model of the pelvic viscera. Sixty-three participants agreed to complete the "body knowledge" survey. The inclusion criteria were that participants were willing to take part and able to read and understand the material that formed part of the questionnaire, any who could not meet these criteria were excluded from the study. Participants were given a questionnaire with two parts. Part 1 assessed their knowledge of the human body; this part of the questionnaire consisted of an image of the human body alongside letters corresponding to common structures within the human body. The participants were asked to place the correct letter onto the body in the place where they considered the organ to be located. Participants were asked to place the following on the body template (Supplementary Material Appendix 1): brain, cornea, lungs, liver, diaphragm, heart, stomach, appendix, bladder, kidneys, pancreas, gallbladder, spleen, adrenals, thyroid, hamstrings, biceps, triceps, quadriceps, cruciate ligament, Achilles tendon. The authors chose the structures based on what appears to come up in everyday life, sports, TV shows and commercials - things that most people will have encountered. The muscles are those that people frequently discuss in terms of keeping fit and sports injuries seen in professional and amateur sports. The abdominal structures were selected as common structures that individuals are likely to have come across in everyday life, TV programs and are found on basic internet searches for abdominal pain. The Achilles tendon was used as an example of how to complete the diagram and placed on the answer sheet. Part 2 of the questionnaire asked for demographics of the participant; “age”, “gender", "highest academic qualification”, "work in healthcare?” and "any visit to a healthcare professional in the last week?" 
After the event, all answer sheets were read by two anatomists and scored independently for correct placement of organs. Correct location(s) on each participant sheet were determined by placing an "answer" version of the location of the organs within the body. This correct answer version consisted of an acetate print out of the participant "blank canvas" version with the organs drawn onto it by a qualified anatomist. A placement by a participant was deemed to be correct if the letter was more than $50 \%$ contained within the boundary of the "answer version". The study was approved by Faculty of Health and Medicine Research Ethics Committee, Lancaster University.

The data were analyzed using a mixed effects logistic model implemented in the R statistical software (R Foundation for Statistical Computing, Vienna, Austria), with subject as a random effect and fixed effects as follows: type of structure $(0=$ internal organ, $1=$ muscle $)$; gender $(0=$ F, $1=\mathrm{M})$; health-related employment $(0=$ no, $1=$ yes $)$; visit to health professional within previous week $(0=$ no, $1=$ yes $)$, highest educational qualification (none, GCSE, A-level or equivalent, university degree); and a quadratic function of age in years. This mixed effects model allowed for unexplained variation in different subjects' ability to give correct answers (random effects) when drawing inferences about the effect of subject-level covariates (fixed effects).

\section{RESULTS}

The "human body" event was not a formal educational intervention with a follow-up summative assessment. Rather, the informal setting and open forum platform served as an opportunity to ask the researchers about the anatomy of the human body. The participants were from a group of sixty three whose mean age was 36.5 years (SD 16.4, range 8-74), their gender comprised 36 
females, 26 males and 1 individual who preferred not to disclose their gender and was excluded from the gender analysis.

Figure 1 shows the number of correct answers for each organ, by gender. The only organ which $100 \%$ of respondents answered correctly was the brain. The biceps muscle and the cornea were the next most correctly answered structures. At the other end of the spectrum the adrenal glands were the structures that fewest respondents were able to identify; less than $15 \%$ of people knew where they were located, with many believing them to be located in the neck. The spleen was the next most weakly identified structure, with $20 \%$ answering correctly closely followed by the gallbladder with only $25 \%$ of respondents being correct. All of the structures in the questionnaire contributed to the reliability, with Cronbach's $\alpha$ of 0.724 , this increased slightly with the removal of the bladder $(0.745)$ or heart $(0.726)$.

The number of correct answers (out of 20) ranged from 2 to 20 and showed a weak, but statistically significant $(\mathrm{p}=0.029)$ quadratic trend with age (Figure 2). Subjects in health-related employment scored significantly higher than those not in health-related employment $(\mathrm{p}<0.001$, odds ratio 3.296). There was a significant interaction between gender and organ type $(\mathrm{p}=0.002)$ with males scoring higher than females in identifying muscles, but not in identifying internal organs. There were no significant associations with highest educational qualification $(p=0.476)$ or visit to health professional within previous week $(\mathrm{p}=0.254)$. Parameter estimates and standard errors for the final model are shown in Table 1. The estimates seen here represent the 
change in log odds of the outcome of difference between the two levels of the factor of interest (i.e. the respondent's ability to answer correctly). The coefficients for age and age squared together describe how the ability to answer correctly varies with age, initially rising with age but then falling (Figure 2).

\section{DISCUSSION}

The value of the current study is twofold. Firstly, it demonstrates the general public's eagerness to learn anatomy. Secondly, it highlights the varied level of anatomical knowledge of the public. The latter aspect emphasizes the value of health education for the public. The second benefit also offers a reminder of the need for anatomical knowledge and literacy as focal points in UK and wider undergraduate medical education. Whilst many of the public do not have or need formal anatomical knowledge, it is beneficial in monitoring and explaining their own health. This study demonstrates that the general population has a wide and varied knowledge about the human body that increases with age. The level of academic qualification does not correlate with ability to identify structures. This work adds to the hypothesis of previous work that has already shown that there are disparities in the ability of the general population and patients in identifying structures, even those with organ or structure specific disorders (Rashid and Jagger, 1996, Weinman et al., 2009; Ramanayake et al., 2014). Health campaigns that specifically target certain diseases or disorders, although they have shown increased referral rates (Meecham et al., 2016) they may still be missing people due to weak anatomical knowledge of whether they have that organ or where it is? In our study many internal organs were poorly identified and individuals who had visited a healthcare professional prior to the quiz fared no better than those who hadn't. This suggests that "Dr Google" may not have all the answers and having visited a 
healthcare professional in the previous week did not help them with their anatomical knowledge. Furthermore this indicates that tasks as simple as identifying organs and structures on the correct side of the body is not straightforward for the general public, hence a clear and competent knowledge of basic anatomy is a fundamental requirement for modern healthcare professionals., This study went one step further in assessing knowledge than other already published studies in that current participants were not given a multiple choice option of locations, they were given a blank canvas in order to try and place the correct location, making it much harder to answer correctly. The work undertaken in this manuscript also represents a more complete assessment of the knowledge of the public, as it has been shown that multiple choice testing allows reactivation of marginal knowledge (Brame and Biel, 2015). The anatomical understanding and knowledge used by medical professionals is learnt over many years and applied in practice long before practitioners are able to interact safely with patients.

A positive performance in respondents who indicated they worked in the broad field of healthcare fared better than those who didn't. The bracket of "worked in healthcare" covers a huge number of professions, from surgeons through to pharmacists and healthcare assistants; regardless of their actual profession, their exposure to the human body and anatomy would be expected to raise their knowledge level above that of the general public; something that the public relies on. The volume of anatomy and physiology and how to apply them are learned over many years, with these subjects often forming core components of their curricula (Kaveevivitchai et al., 2009). Evidence points towards improved consultation and patient satisfaction when doctors and patients have a shared anatomical/medical vocabulary (Williams and Ogden, 2004). It is reassuring that these results support an improved performance in individuals who work in healthcare (Spielmann and Oliver, 2005, Gupta et al., 2008, Kong et al., 
2015), this is not always the case, a small number of studies have shown a lack of distinction in performance; established doctors showing less anatomical knowledge than medical students in certain areas of medicine and anatomy (Segaren et al., 2012).

It is well known that ageing increases the likelihood of diseases and disorders and that these patients will have consulted medical professionals on a more regular basis (Burkle et al., 2007; Akushevich et al., 2013). These patients will likely also have explored internet-based resources and therefore may be familiar with the location of the structure that is causing them pain (Jeannot et al., 2004). This may explain the average higher number of correct answers in the $40-49$ age bracket than those from either side, as data from the United Kingdom show that the number of consultation rates per person begins to increase in the 40s (Ackermann Rau et al., 2014).Furthermore, this age group may become more aware of the location of structures and organs that cause conditions that necessitate an understanding of where these structures are. Weinman and colleagues (2009) documented similar findings in their study; participants from specific diagnostic groups, liver disease and diabetes, were more accurate in identifying the organs involved (Weinman et al., 2009).

It has also been argued that individuals with lower levels of education need special skills and support for understanding health-related information (Hippisley-Cox and Vinogradova, 2009). The data presented here do not support the hypothesis that those with fewer or lower educational qualifications perform significantly worse than others, although the individuals with the lowest scores were from this demographic profile. This study is the first, to the authors knowledge, that looks at the performance of individuals under the age of 18 . The youngest respondents were aged $8,8,11,11,12$ and 17 answered $9,3,10,6,2$ and 10 out of 20 correct, respectively. The age of many of the under 18 respondents places them within the "pre-adolescent" age bracket. It has 
been demonstrated that these students show a desire to learn about the human body, but may be limited initially due to the lack of knowledge of relevant content from their educators (Burns, 2008). Educational outreach to students of this age has a two-fold benefit 1) they have a desire to learn given the anatomical and physiological changes that are occurring as they grow and move through puberty. 2) They are at an age where experimentation with substances such as tobacco becomes likely. Educational outreach which combines normal anatomy and pathological changes that result from life choices is likely to be more beneficial at this age and linked to improved health outcomes in later life (Burns, 2012). Innovative and highly successful outreach programs can be utilized to identify the individual needs of students and the staff educating them, using a variety of settings which provide the scope for better educational and health outcomes (Burns, 2002). The role of Universities in providing outreach education in this field suggests that there are limited knowledge and opportunities within core educational curricular. In the UK there are a variety of providers that offer secondary educational courses as part of GCSE and A-Level qualifications. However, the amount of anatomy in them is very limited. The UK Government documents give very little information about what specifics linked to anatomical structures are taught; rather they are somewhat vague with only direct reference to the heart in its physiological role, instead focusing heavily on cellular structure and function(s) (Department for Education, 2014). An improvement in delivery of basic anatomical knowledge within primary and secondary education may have beneficial health outcomes in later life, as well as reducing burden on healthcare services by ensuring competent communication between patient and healthcare professional.

\section{Study limitations}


This pilot observational cross sectional study employed a small sample size compared to similar publications (Weinman et al., 2009; Ramanayake et al., 2014). The authors did not explore specific diagnostic groups such as diabetes, cardiovascular disorders and liver disease. The choice of individuals to participate was interpreted by researchers as them doing so through their own interest in anatomy and health and thus may represent a biased population that are at the more informed end of the general public spectrum of knowledge. Similarly, it cannot be compared how the demographics of the participants are representative of the other attendees on the day, as these data were not collected.

This study is also England/UK-centric and may not be representative of the knowledge of other areas of the world where different educational content as part of compulsory education or prevalence of different diseases that affect the population may lead to a different level of knowledge of a given area.

Finally, Lancaster is one of western Lancashire's major healthcare hubs and this explains the high proportion of healthcare providers in this study. However, the value of this possible skewed representation of the general population of Lancaster highlights the need to strengthen the existing anatomical literacy.

\section{CONCLUSIONS}

Health agencies such as the NHS are successfully working on improving the general public's understanding of health and wellbeing. Advertising campaigns for health screening related to structures such as the prostate and abdominal aorta, see increased referral rates, but little is known about whether individuals actually know where these structures are within their own body (Meecham et al., 2016). Body knowledge of the general public appears to vary greatly and this 
brings into question their individual decision-making about their own care. Effective communication between doctor and patient and a matched vocabulary significantly impacts the efficiency of a consultation as well as the patient compliance (Williams and Ogden, 2004). The data gathered suggest that there are gender differences in being able to identify internal organs versus muscles. The type-by-gender interaction is significantly positive, which is explained by the fact that the success rate for males identifying muscles is significantly higher than all three other categories, i.e. males and females are more-or-less equally good at identifying internal organs, but males are better than females at identifying muscles. This information, along with recent data showing how functional brain imaging predicts the success of public health campaigns (Falk et al., 2016), should be considered by medical students, health care professionals and public health campaigners when consulting with patients and designing health literature and education materials. Future research is needed to determine the expected anatomical literacy of the general public.

The current study extends beyond data collection and points towards a possible mode of public engagement in anatomy. An event such as the Lancaster University's CITC, involving undergraduate students as facilitators, has the potential to highlight the existing gaps in health literacy and to form a bridge between healthcare professionals and patients (Chinn, 2011). An additional advantage relates to the bioethical considerations when using human or cadaveric material in public engagement (Myser, 2007). The sometimes understated value of anatomical models circumvents such issues. Technological advances in the computerized representation of the human body further has become more realistic and serves as an alternative to controversial use of human material in the public domain (Murgitroyd et al., 2015). Finally, medical students 
should be reminded that their anatomical knowledge should be better than that of their patients, and the best place to begin to learn this is in Medical School. 


\section{ACKNOWLEDGEMENTS}

The authors would like to thank all the members of the public who kindly completed the questionnaires and "The Campus in the City" team for the opportunity to run the human body event. 


\section{NOTES ON CONTRIBUTORS:}

ADAM M TAYLOR, Ph.D., B.Sc. (Hons.), S.F.H.E.A, is a senior lecturer in anatomy and Director of the Clinical Anatomy Learning Centre, in Lancaster Medical School, Faculty of Health and Medicine, at Lancaster University, Lancaster, UK. He teaches embryology, microscopic and gross anatomy to first-, second- and third-year medical and biomedical students. His research interests include rheumatology, microscopic anatomy, gross anatomy, medical education, and medical history.

PETER J DIGGLE, PhD, M.Sc., B.Sc. (Hons.), is a distinguished professor in CHICAS (Centre for Health Informatics, Computing and Statistics) in Lancaster Medical School, Faculty of Health and Medicine, at Lancaster University, Lancaster, UK. He teaches statistical methods for biomedical and public health applications to postgraduate students. His research interests include longitudinal data analysis, spatial statistics, environmental and tropical disease epidemiology and health surveillance.

QUENTON WESSELS, Ph.D., M.Sc. (Clin.Educ.), F.H.E.A., is a senior lecturer in the Department of Anatomy, School of Medicine, University of Namibia, Windhoek, Namibia He teaches embryology and microscopic and gross anatomy to first- and second-year medical and pharmacy students. His research interests include microscopic anatomy, gross anatomy, medical education, and medical history. 


\section{LITERATURE CITED}

Ackermann Rau S, Sakarya S, Abel T. 2014. When to see a doctor for common health problems: Distribution patterns of functional health literacy across migrant populations in Switzerland. Int $\mathbf{J}$ Public Health 59:967-974.

Akushevich I, Kravchenko J, Ukraintseva S, Arbeev K, Yashin AI. 2013. Time trends of incidence of age-associated diseases in the US elderly population: Medicare-based analysis. Age Ageing 42:494-500.

Baram-Tsabari A, Yarden A. 2007. Interest in biology: A developmental shift characterized using self-generated questions. Am Biol Teach 69:532-540.

Bates AW. 2008. "Indecent and demoralising representations": Public anatomy museums in midVictorian England. Med Hist 52:1-22.

Bernhard JC, Isotani S, Matsugasumi T, Duddalwar V, Hung AJ, Suer E, Baco E, Satkunasivam R, Djaladat H, Metcalfe C, Hu B, Wong K, Park D, Nguyen M, Hwang D, Bazargani ST, de Castro Abreu AL, Aron M, Ukimura O, Gill IS. 2016. Personalized 3D printed model of kidney and tumor anatomy: A useful tool for patient education. World J Urol 34:337-345.

Brame CJ, Biel R. 2015. Test-enhanced learning: The potential for testing to promote greater learning in undergraduate science courses. CBE Life Sci Educ 14:es4. 
Burns ER. 2002. Anatomy of a successful K-12 educational outreach program in the health sciences: Eleven years experience at one medical sciences campus. Anat Rec 269:181-193.

Burns ER. 2008. Functional anatomy of the cardiovascular system: Professional development for PreK-3 teachers using a "train and equip" method results in learning opportunities for students. Anat Sci Educ 1:119-125.

Burns ER. 2012. Healthy Lungs: Cancer education for middle school teachers using a "train and equip" method. J Cancer Educ 27:179-185.

Bürkle A, Caselli G, Franceschi C, Mariani E, Sansoni P, Santoni A, Vecchio G, Witkowski JM, Caruso C. 2007. Pathophysiology of ageing, longevity and age related diseases. Immun Ageing $4: 4$.

Chinn D. 2011. Critical health literacy: A review and critical analysis. Soc Sci Med 73:60-67.

Department for Education. 2014. GCE AS and A Level Subject Content for Biology, Chemistry, Physics and Psychology. $1^{\text {st }}$ Ed. London, UK: Department of Education. 41 p. URL: https://www.gov.uk/government/uploads/system/uploads/attachment_data/file/593849/Science AS_and_level_formatted.pdf [accessed 23 August 2017]. 
Drake RL, McBride JM, Lachman N, Pawlina W. 2009. Medical education in the anatomical sciences: The winds of change continue to blow. Anat Sci Educ 2:253-259.

Falk EB, O'Donnell MB, Tompson S, Gonzalez R, Dal Cin S, Strecher V, Cummings KM, An L. 2016. Functional brain imaging predicts public health campaign success. Soc Cogn Affect Neurosci 11:204-214.

Ferrari G. 1987. Public anatomy lessons and the carnival: The anatomy theatre of Bologna. Past Present 117:50-106.

Fiore N. 2016. Prostate cancer. Nurs Stand 30:17.

Fonseca GR, Finn GM. 2016. The impact of body worlds on adult visitors' knowledge on human anatomy: A preliminary study. Clin Anat 29:439-445.

GMC. 2009. General Medical Council. Tomorrow’s Doctors: Outcomes and Standards, for Undergraduate Medical Education. Regulating Doctors, Ensuring Good Medical Practice. 3rd Ed. London, UK: General Medical Council. 104 p.

GMC. 2015. General Medical Council. Outcomes for Graduates (Tomorrow's Doctors) $1^{\text {st }}$ Ed. Manchester, UK: General Medical Council. 20 p.

Gillingwater TH, Findlater GS. 2015. Anatomy: Back in the public spotlight. Lancet 385:1825. 
Goeller A. 2007. Interior landscapes: Anatomy art and the work of Gunther Von Hagens. Genus Gend Mod Cult 9:271-289.

Gosh SK. 2015. Human cadaveric dissection: A historical account from ancient Greece to the modern era. Anat Cell Biol 48:153-169.

Gupta Y, Morgan M, Singh A, Ellis H. 2008. Junior doctors' knowledge of applied clinical anatomy. Clin Anat. 21:334-338.

Hippisley-Cox J, Vinogradova Y. 2009. Trends in Consultation Rates in General Practice 1995 to 2008: Analysis of the QResearch ${ }^{\circledR}$ Database. $1^{\text {st }}$ Ed. Leeds, West Yorkshire, UK: QResearch and NHS Health and Social Care Information Centre. 23 p. URL:

http://content.digital.nhs.uk/catalogue/PUB01077/tren-cons-rate-gene-prac-95-09-95-08-rep.pdf [accessed 11May 2017].

Jeannot JG, Froehlich F, Wietlisbach V, Burnand B, Terraz O, Vader JP. 2004. Patient use of the Internet for health care information in Switzerland. Swiss Med Wkly 134:307-312.

Jones DG. 2002. Re-inventing anatomy: The impact of plastination on how we see the human body. Clin Anat 15:436-440. 
Kaveevivitchai C, Chuengkriankrai B, Luecha Y, Thanooruk R, Panijpan B, Ruenwongsa P. 2009. Enhancing nursing students' skills in vital signs assessment by using multimedia computerassisted learning with integrated content of anatomy and physiology. Nurse Educ Today 29:6572.

Kelly PA, Haidet P. 2007. Physician overestimation of patient literacy: A potential source of health care disparities. Pat Educ Couns 66:119-122.

Kong VY, Oosthuizen GV, Sartorius B, Keene CM. Clarke DL. 2015. Correlation between ATLS training and junior doctors' anatomical knowledge of intercostal chest drain insertion. J Surg Educ 72:600-605.

Kripalani S, Weiss BD. 2006. Teaching about health literacy and clear communication. J Gen Intern Med 21:888-890.

Lerner EB, Jehle DV, Janicke DM, Moscati RM. 2000. Medical communication: Do our patients understand? Am J Emerg Med 18:764-766.

Meecham L, Jacomelli J, Pherwani AD, Earnshaw J. 2016. Self-referral to the NHS abdominal aortic aneurysm screening programme. Eur J Vasc Endovasc Surg 52:317-321.

Morriss-Kay G. 2002. Review. J Anat 200:535-536. 
Murgitroyd E, Madurska M, Gonzalez J, Watson A. 2015. 3D digital anatomy modelling Practical or pretty? Surgeon 13:177-180.

Myser C. 2007. Taking public education seriously: BODY WORLDS, the science museum, and democratizing bioethics education. Am J Bioeth 7:34-36.

Nielsen-Bohlman L, Panzer AM, Kindig DA (Editors). 2004. Health Literacy: A Prescription to End Confusion. $1^{\text {st }}$ Ed. Washington, DC: National Academies Press. 345 p.

Ramanayake BS, Liyanagoda NE, Dahanayake PK. 2014. Do patients understand medical communication? Patients' knowledge on anatomical location of organs in human body. Sci Res J 2:12-16.

Rashid A, Jagger C. 1996. Patients' knowledge of anatomical location of major organs within the human body: A comparison between Asians and non-Asians. Fam Pract 13:450-454.

Segaren N, Vaghela K, Markar S, Gilleard O, Segaren N, Shanmugarajah K. 2012. The anatomical knowledge of healthcare professionals referring to a hand trauma centre. Int J Surg 10:S79.

Smith CF, McManus B. 2015. The integrated anatomy practical paper: A robust assessment method for anatomy education today. Anat Sci Educ 8:63-73. 
Spielmann, PM, Oliver CW. 2005. The carpal bones: A basic test of medical students' and junior doctors' knowledge of anatomy. Surgeon 3:257-259.

Weinman J, Yusuf G, Berks R, Rayner S, Petrie KJ. 2009. How accurate is patients' anatomical knowledge: A cross-sectional, questionnaire study of six patient groups and a general public sample. BMC Fam Pract 10:43.

Whitehead D. 2004. Health promotion and health education: Advancing the concepts. J Adv Nurs 37:311-320.

Williams MV, Baker DW, Parker RM, Nurss JR. 1998. Relationship of functional health literacy to patients' knowledge of their chronic disease. A study of patients with hypertension and diabetes. Arch Intern Med 158:166-172.

Williams N, Ogden J. 2004. The impact of matching the patient's vocabulary: A randomized control trial. Fam Pract 21:630-635.

Wormald BW, Schoeman S, Somasunderam A, Penn M. 2009. Assessment drives learning: An unavoidable truth? Anat Sci Educ 2:199-204. 


\section{FIGURE LEGENDS}

Figure 1: A graph showing the number of the correct answers by structure for males (blue) and females (red) as percentage of correct responses. The brain was answered correctly by $100 \%$ of respondents. The adrenals, spleen and gallbladder were the structures which were identified most poorly by respondents.

Figure 2. Scores (percentage of correct answers out of 20, solid dots) against age in years, with modelled relationship between score and age (black solid curve). Gender was not significantly associated with score, but is indicated for completeness (blue for Females, red for Males).

Table 1: Parameter estimates (log-odds ratio scale) and standard errors (in parentheses) for the fitted logistic model. For binary factors (e.g., gender or organ type) each estimate represents the change in log odds of the outcome (i.e., the respondent's ability to answer correctly) between the two levels of the factor. The coefficients for age and age squared together describe how the ability to answer correctly varies with age, initially rising with age but then falling (Figure 2). 


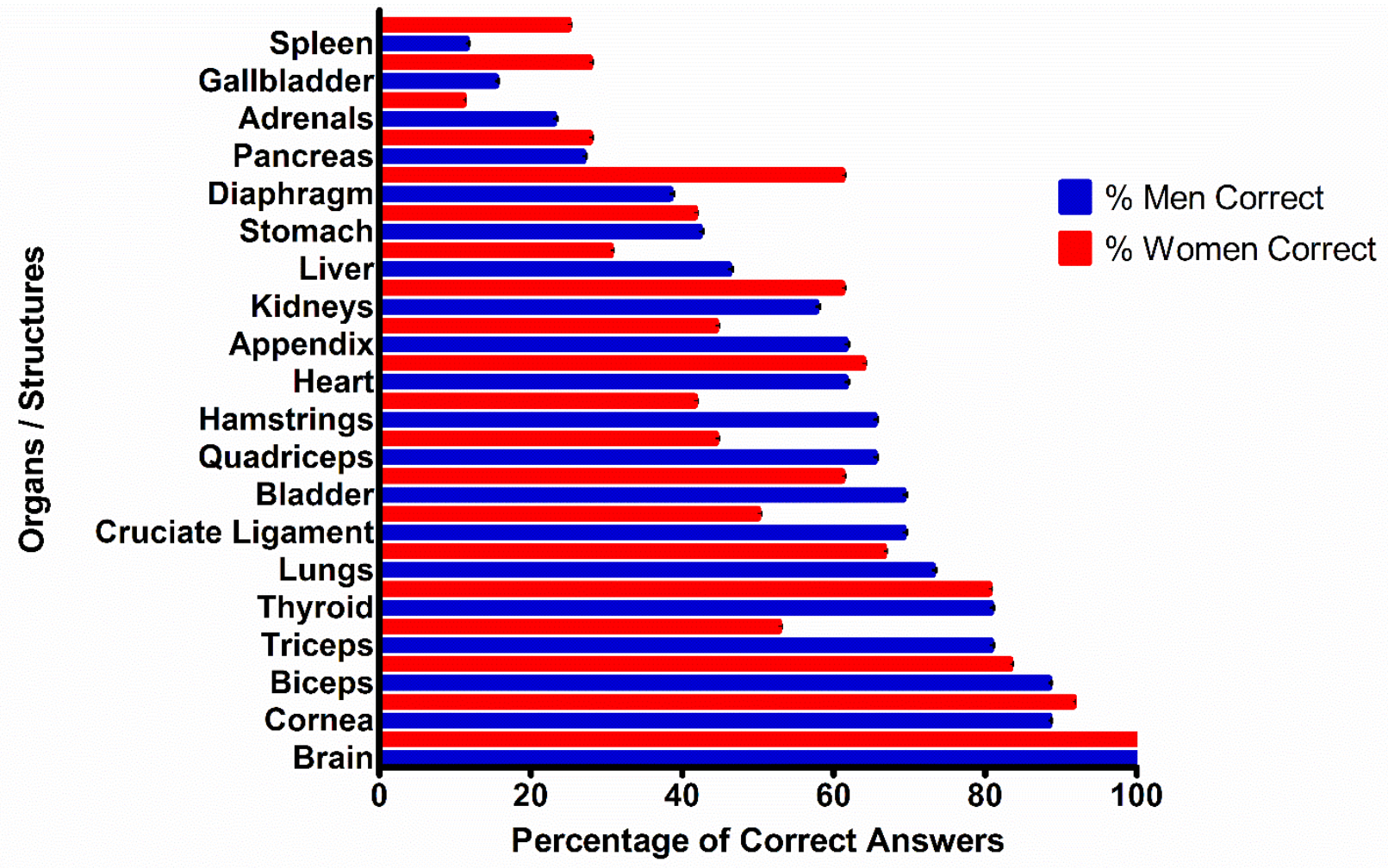

Figure 1 


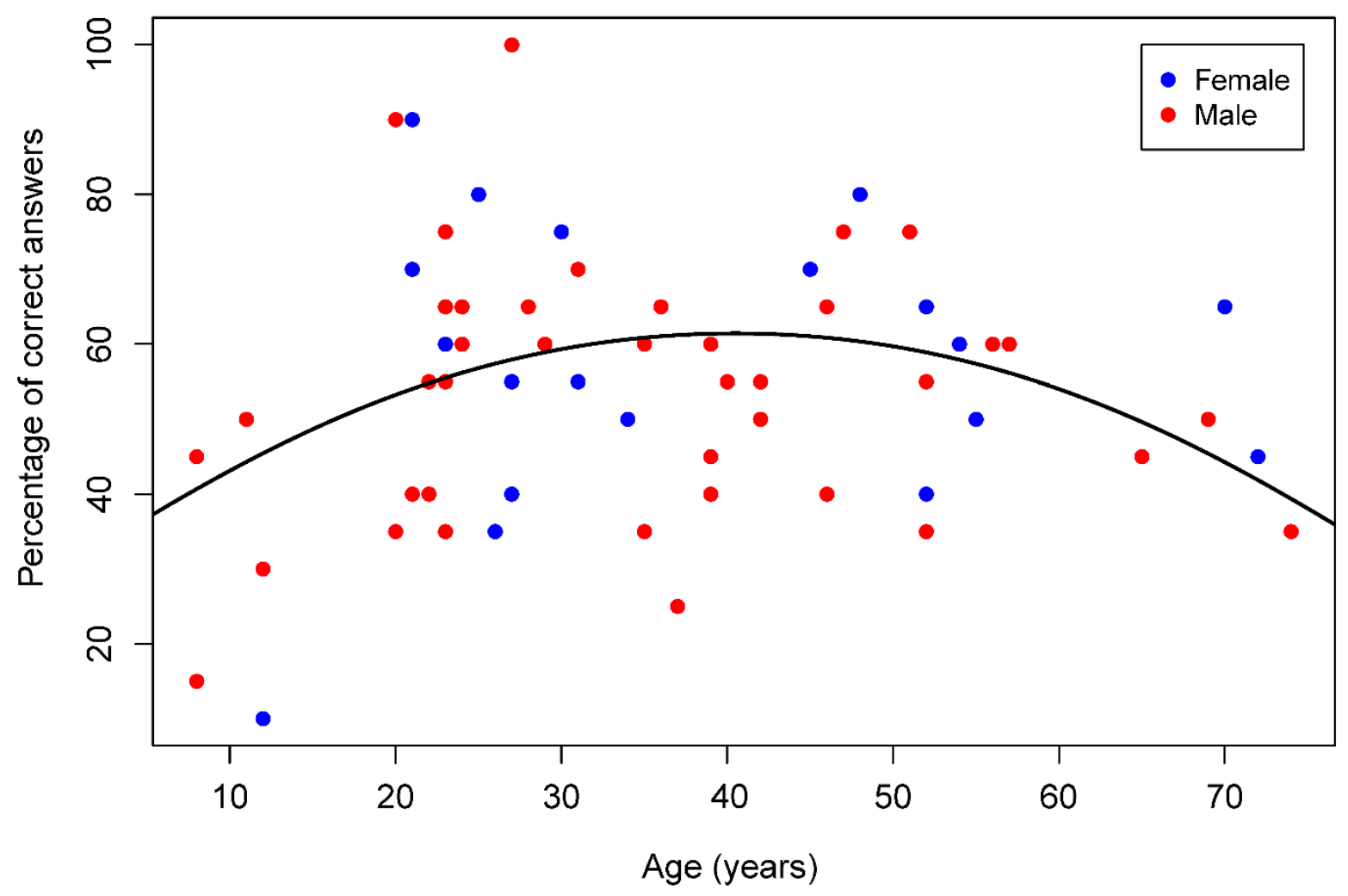

Figure 2 


\begin{tabular}{|l|l|}
\hline Effect & Estimate (standard error) \\
\hline age & $0.092(0.057)$ \\
\hline age-squared & $-0.076(0.029)$ \\
\hline gender & $0.112(0.189)$ \\
\hline organ type & $0.066(0.181)$ \\
\hline gender-by-type interaction & $0.916(0.294)$ \\
\hline health-related employment & $1.193(0.297)$ \\
\hline
\end{tabular}

Table 1: Parameter estimates (log-odds ratio scale) and standard errors (in parentheses) for the fitted logistic model. For binary factors (eg gender or organ type) each estimate represents the change in log odds of the outcome (i.e. the respondent's ability to answer correctly) between the two levels of the factor. The coefficients for age and age squared together describe how the ability to answer correctly varies with age, initially rising with age but then falling (Figure 2). 